\title{
A fire station-based dietary and lifestyle, controlled intervention for UK firefighter health
}

\author{
G.R. Lessons and D. Bhakta \\ Public Health Nutrition Research Group, London Metropolitan University, London, N7 8DB, UK
}

The overweight and obesity epidemic has not avoided the fire service, with prevalence exceeding that of the UK general population ${ }^{(1,2)}$. Furthermore, firefighters are at increased risk of acute myocardial infarction (MI) from exposures including intense heat which can impair vascular function ${ }^{(3)}$, and an obesogenic environment identified as being characteristic of fire stations ${ }^{(2)}$. Faced with this complex combination of exposures, it is critical that the modifiable risk factors of MI are addressed. The UK's first fire station-based dietary and lifestyle intervention pilot trial indicated that a low burden, low intensity fire station intervention was highly feasible, could significantly improve dietary behaviour and result in significantly improved body composition of firefighters (2). Limitations of the pilot included a small sample size, its brief duration (one month), the absence of a control group for measuring dietary intake, and statistical tests which were not able to analyse the interaction between time and group. Questions therefore remained in terms of scalability, durability and efficacy, therefore further research was necessary.

This was a prospective cluster-controlled trial comprising 89 full-time firefighters (intervention: $n=45$, control: $n=44$ ) from twelve London fire stations (seven intervention, five control), recruited in 2019. Outcome measures (measured at baseline and four-month follow-up) were: Dietary intake measured by a food frequency questionnaire validated for UK firefighters; Physical activity assessed by the Concise Physical Activity Questionnaire ${ }^{(4)}$; Mood and energy level measured by a 10-point lickert scale; Total adiposity and skeletal muscle mass (SMM) measured via bioelectrical impedance analysis utilising the Tanita MC-780 system; Central adiposity measured by waist circumference (WC); Body weight and body mass index (BMI). The intervention comprised a thirty-minute nutrition and health PowerPoint presentation (group education) and a twenty-minute face-to-face personalised nutrition consultation. Intervention content focused on portion control strategies, promotion of the Mediterranean diet, environmental modification, and education regarding risk factors for obesity-related non-communicable diseases. Mixed model ANOVAs were used to identify changes over time and between groups.

The intervention elicited significant $(p<0.01)$ improvements in mean intake of energy $(-244 \mathrm{kcal})$, total fat $(-12 \mathrm{~g})$, saturated fatty acids $(-12 \mathrm{~g})$, sodium $(-311 \mathrm{mg})$, sugars, preserves and snacks $(-19 \mathrm{~g})$, and mean improvements in fat mass $(-2 \mathrm{~kg})$, percentage body fat $(-1.7 \%)$, WC $(-1.7 \mathrm{~cm})$, weight $(-1.7 \mathrm{~kg})$, BMI $(-0.5 \mathrm{~kg} / \mathrm{m} 2)$, mood $(+0.9)$ and energy level $(+1.2)$ compared with the control group increasing in WC $(+1.9 \mathrm{~cm})$. No significant changes were observed in SMM or physical activity $(p>0.01)$.

This study has shown that this low-cost, low-burden and low-intensity fire station dietary and lifestyle intervention comprising group education, environmental modification and personalised nutrition is effective for improving dietary behaviour, body composition and indicators of mental health. The intervention's high level of feasibility renders it appropriate for organisational rollout.

\section{References}

1. Munir, F, Clemes, S, Houdmont, J et al. (2012) Occup Med 62(5), 362-365.

2. Lessons, GR, Bhakta, D (2018) Proc Nut Soc 77(OCE1), E21.

3. Hunter, AL, Shah, ASV, Langrish, JP et al. (2017) Circulation 135, 1284-1295.

4. Sliter, KA, Sliter, MT (2014) Int J Stress Manag, 21(4), 348-360 